\title{
Podocalyxin promotes glioblastoma multiforme cell invasion and proliferation by inhibiting angiotensin-(1-7)/Mas signaling
}

\author{
BO LIU, YU LIU and YUGANG JIANG
}

\begin{abstract}
Department of Neurosurgery, The Second Xiangya Hospital, Central South University, Changsha, Hunan 410011, P.R. China
\end{abstract}
Received October 13, 2014; Accepted January 12, 2015

DOI: $10.3892 /$ or.2015.3813

\begin{abstract}
Podocalyxin (PODX) reportedly enhances invasion in many human cancers including glioblastoma multiforme (GBM). Recent studies have shown that the local renin-angiotensin system (RAS) in tumor environment contributes significantly to tumor progression. As a counterregulatory axis in RAS, angiotensin (Ang)-(1-7)/Mas signaling has been shown to inhibit the growth and invasiveness of several human cancers including GBM. In the present study, we examined the crosstalk between PODX and Ang-(1-7)/Mas signaling in GBM cells, and assessed its impact on GBM cell invasion and proliferation. A strong negative correlation between the expression of PODX and Mas in GBM tumor tissues from 10 consecutive patients $(\mathrm{r}=-0.768, \mathrm{p}<0.01)$ was observed. The stable overexpression of PODX in LN-229 and U-118 MG human GBM cells decreased the expression of Mas at the mRNA and protein levels, which led to decreased density of Ang-(1-7)-binding Mas on the cell membrane. This effect was completely abolished by selective phosphatidylinositol 3-kinase (PI3K) inhibitor BKM120. By contrast, the stable knockdown of PODX in LN-229 and U-118 MG cells increased the expression of Mas and the density of Ang-(1-7)-binding Mas on the cell membrane. Overexpression and knockdown of PODX respectively reversed and enhanced the inhibitory effects of Ang-(1-7) on the expression/activity of matrix metalloproteinase-9 and cell invasion and proliferation in GBM cells. Although the overexpression of Mas showed no significant effect on the promoting effect of PODX on GBM cell invasion and proliferation in the absence of Ang-(1-7), it completely eliminated the effect of PODX in the presence of Ang-(1-7). In conclusion, to the best of our knowledge, the present study provided the first evidence that PODX inhibits Ang-(1-7)/Mas signaling by downregulating the expression of
\end{abstract}

Correspondence to: Dr Yugang Jiang, Department of Neurosurgery, The Second Xiangya Hospital, Central South University, 139 Middle Renmin Road, Changsha, Hunan 410011, P.R. China

E-mail: yugang_jiang@163.com

Key words: podocalyxin, angiotensin-(1-7), Mas, glioblastoma multiforme, invasion, proliferation, phosphatidylinositol 3-kinase, matrix metalloproteinase-9
Mas through a PI3K-dependent mechanism in GBM cells. This effect led to enhanced GBM cell invasion and proliferation. The results of this study add new insight into the biological functions of PODX and the molecular mechanisms underlying GBM progression.

\section{Introduction}

Glioblastoma (GBM) is the most common and most malignant primary adult brain tumor [World Health Organization (WHO) grade IV] (1). Despite great advances in surgery, chemotherapy and radiotherapy, the median survival is only 12-15 months for patients with GBMs (2). This poor outcome renders GBM an urgent subject of investigation. The poor prognosis of GBM is largely attributed to rapid growth, invasiion and migration, and a high rate of recurrence (3). The highly invasive nature of GBM makes surgical resection non-curative, and it has been proposed that invading cells may be more resistant to radiation and chemotherapy (3). Therefore, it is important to identify and confirm potential therapeutic targets involved in the invasion and progression of GBM.

Podocalyxin (PODX) is a highly glycosylated and sialylated transmembrane protein, and a CD34 ortholog normally expressed on hematopoietic stem cells, hemangioblasts, vascular endothelial cells, podocytes and a subset of neural progenitors (4). Aberrant PODX expression has been reported in leukaemia, undifferentiated thyroid and renal cell carcinoma (5-8). High levels of PODX protein expression have been correlated with poor outcome in a subset of breast carcinomas and have also been associated with increased aggressiveness of breast and prostate cancer cells $(9,10)$. A recent report has shown that PODX promotes astrocytoma cell invasion and survival against apoptotic stress (11), suggesting that PODX also contributes to GBM progression. Although there is evidence that PODX participates in epithelial-mesenchymal transition and interacts with different mediators of metastasis, the role of PODX remains to be elucidated (12).

The renin-angiotensin system (RAS), with its active principle angiotensin II (Ang II), plays a fundamental role in the regulation of blood pressure and fluid homeostasis in mammals (13). Recent experimental and clinical evidence have indicated that, in addition to systemically produced angiotensin, the tumor environment contains RAS components necessary to produce angiotensin locally, and the local RAS system contributes significantly to tumor progres- 
sion (14). Changes in the expression of RAS components have been associated with the increased capacity of transformed cells to invade and metastasize (15). For example, in gastric cancer, expression of angiotensin-converting enzyme (ACE) and angiotensin receptor 1 (AT1R) in the tumor influences metastatic behavior (16). In addition to the ACE/Ang II/AT1 receptor axis, the RAS possesses a counter-regulatory axis composed of ACE2, Ang-(1-7) and the Mas receptor (17). Ang-(1-7) is recognized as a critical component of the RAS, as it exerts various actions, many of which are opposite to those of Ang II (18). Ang-(1-7) can be generated directly from Ang II by ACE2 with high efficiency $(18,19)$, and directly from Ang I by neutral endopeptidase and prolylendopeptidase (20). It has been established that the $G$ protein-coupled receptor Mas is a functional binding site for Ang-(1-7) $(21,22)$. It has been reported that Ang-(1-7) inhibits the growth and invasiveness of human lung cancer cells through activation of Mas (14). Ang-(1-7) has been shown to markedly decrease the proliferation of human GBM cells through Mas both in vitro and in vivo (23).

PODX and the Ang-(1-7)/Mas axis play important roles in GBM progression. Our pilot study suggested that PODX downregulates Mas expression in GBM cells. To the best of our knowledge, in the present study, we examiend for the first time the effect of PODX on Mas expression and Ang-(1-7)/ Mas signaling in GBM cells, and assessed its impact on GBM cell invasion and proliferation.

\section{Materials and methods}

Cell lines and reagents. LN-229 (CRL-2611) and U-118 MG (HTB-15) human GBM cell lines were purchased from the American Type Culture Collection (Manassas, VA, USA). Human full-length PODX cDNA was subcloned into the pcDNA 3.1 expression vector. Human PODX shRNA plasmid (RHS3979-98487921) was purchased from Open Biosystems (Huntsville, AL, USA). Human MAS1 cDNA clone (SC118704) was purchased from OriGene (Beijing, China) and the full-length Mas cDNA sequence was subcloned into the pIRESpuro3 plasmid (Clontech, Mountain View, CA, USA) to generate a Mas expression vector. Mouse monoclonal antiPODX (3D3) (39-3800) antibody and Lipofectamine 2000 transfection reagent were purchased from Life Technologies (Carlsbad, CA, USA). Selective phosphatidylinositol 3-kinase (PI3K) inhibitor BKM120 (sc-364437A), mouse monoclonal anti-MAS1 (G-1) (sc-390453) antibody, mouse monoclonal anti-matrix metalloproteinase-9 (MMP-9) (2C3) (sc-21733), and goat polyclonal anti-glyceraldehyde-3-phosphate dehydrogenase (GAPDH) (V-18) (sc-20357) antibodies were purchased from Santa Cruz Biotechnology, Inc. (Santa Cruz, CA, USA). The QCM ECMatrix 24-well $(8 \mu \mathrm{M})$ Fluorimetric Cell Invasion Assay kit (ECM554) was purchased from Chemicon (Millipore, Billerica, CA, USA). The SensoLyte 520 MMP-9 assay kit (no. 71155) was purchased from AnaSpec (Fremont, CA, USA). The Methylthiazoletetrazolium (MTT) Cell Proliferation Assay kit (no. 30-1010K) was purchased from the American Type Culture Collection. ${ }^{125}$ I-Sodium iodide (carrier free, $100 \mathrm{mCl} / \mathrm{ml}$ ) was purchased from Amersham Biosciences (Piscataway, NJ, USA). Puromycin, G418 and Ang-(1-7) were purchased from Sigma-Aldrich (St. Louis, MO, USA). Human
GBM tumor tissues were collected from surgery-resected GBM tumors from 10 consecutive patients at the Second Xiangya Hospital, Central South University (Hunan, China) during January-March, 2014.

Transfection and lentiviral transduction. PODX and the Mas expression vectors were transfected into LN-229 and U-118 MG cells using Lipofectamine 2000 transfection reagent (Life Technologies) according to the manufacturer's instructions. Pools of stable transfectants were generated via selection with $\mathrm{G} 418(800 \mu \mathrm{g} / \mathrm{ml})$ and puromycin $(5 \mu \mathrm{g} / \mathrm{ml})$ according to the manufacturer's instructions. Lentiviral transduction was performed in LN-229 and U-118 MG cells. Lentiviral particles were packaged with vectors psPAX2 and pMD2.G according to the manufacturer's instructions (Open Biosystems). A control virus containing a scrambled shRNA sequence that did not lead to specific degradation of any cellular mRNA was used as a negative control for PODX-shRNA lentiviral particles. Pools of stable transductants were generated via selection with puromycin $(5 \mu \mathrm{g} / \mathrm{ml})$.

Western blot analysis. Tissue homogenate or cultured cells was lysed with a hypotonic buffer containing $2 \%$ Nonidet-P and a protease inhibitor cocktail (Sigma) by sonication three times for $3 \mathrm{sec}$ on ice. The supernatant obtained after centrifugation at $2,000 \mathrm{x} \mathrm{g}$ for $15 \mathrm{~min}$ at $4^{\circ} \mathrm{C}$ was used to determine the protein concentration using the Coomassie blue method and for subsequent steps. Equal amount of proteins for each sample was separated by $8-15 \%$ SDS-polyacrylamide gel and blotted onto a polyvinylidene difluoride microporous membrane (Millipore). The membrane was incubated for $1 \mathrm{~h}$ with a 1:1,000 dilution of primary antibody, and then washed and revealed using secondary antibodies with horseradish peroxidase-conjugated $(1: 5,000,1 \mathrm{~h})$. Peroxidase was revealed with a GE Healthcare ECL kit (Shanghai, China). Three independent experiments were performed for each western blot analysis.

Quantitative reverse transcription PCR. RNA was prepared using TRIzol reagent followed by purification with TURBO DNA-free system (Ambion, Austin, TX, USA). The cDNAs were synthesized using SuperScript II reverse transcriptase (Invitrogen). Quantitative PCR was performed on an ABI Prism 7700 Sequence Detection System, with use of the fluorescent dye SYBR-Green Master Mix (Applied Biosystems) according to the manufacturer's instructions. The primers used were: for human Mas: 5'-TTCCGGATGAGAAGAAATCC-3' (forward), and 5'-ATGGCCAGAAGAAAGCTCAT-3' (reverse); for human GAPDH: 5'-GTCAGTGGTGGACCT GACCT-3' (forward), and 5'-TGCTGTAGCCAAATTCG TTG-3' (reverse). The threshold cycle (Ct) for each PCR product was calculated with the instrument's software, and obtained $\mathrm{Ct}$ values were normalized by subtracting the $\mathrm{Ct}$ values of GAPDH. The resulting $\triangle \mathrm{Ct}$ values were then used to calculate the relative change in mRNA expression as a ratio (R) according to the $2^{-\Delta \Delta C t}$ equation method. GAPDH was used as a loading control. Each experiment was repeated three times in duplicate.

[ ${ }^{125}$ I]Ang-(1-7) binding assay. Cells were rinsed twice with RPMI-1640 and equilibrated on ice with incubation buffer 
(RPMI-1640 containing 0.2\% BSA and a protease inhibitor cocktail, pH 7.4) for $30 \mathrm{~min}$. Subsequently, the plates were incubated at $4^{\circ} \mathrm{C}$ for 60 min with incubation buffer containing $0.5 \mathrm{nmol} / \mathrm{l}^{125} \mathrm{I}$-Ang-(1-7) labeled as previously described (24). Incubation was stopped by rinsing the cells three times with ice-cold phosphate-buffered saline (PBS). The cells were solubilized by incubation with $0.1 \mathrm{~mol} / 1 \mathrm{NaOH}$ for $60 \mathrm{~min}$ and the radioactivity was measured. Non-specific binding was determined in the presence of $10 \mu \mathrm{mol} / 1$ unlabeled Ang-(1-7), which was no $>15 \%$. Specific binding was calculated by the subtraction of non-specific binding from total binding. The disintegrations/min (dpm) data were normalized against cell number (per 20,000 cells) and shown as fold-changes to that of control cells (designated as 1). Each experiment was repeated three times in duplicate.

Cell invasion assay. In vitro cell invasion assays were performed with the QCM ECMatrix 24-well $(8-\mu \mathrm{M})$ Fluorimetric Cell Invasion Assay kit (Chemicon; Millipore) according to the manufacturer's instructions $(25,26)$. The kit used an insert polycarbonate membrane with an $8-\mu \mathrm{M}$ pore size. The insert in the invasion kit was coated with a thin layer of ECMatrix. LN-229 and U-118 MG cells with or without Ang-(1-7) (100 nM) treatment were seeded in the insert (the upper chamber) at $5 \times 10^{4}$ cells/well in RPMI-1640 serum-free medium. Complete medium $(600 \mu \mathrm{l})$ with $10 \%$ fetal bovine serum was added to the lower chamber. After $24 \mathrm{~h}$ of incubation, invading cell numbers were determined by running a fluorescent cell dose curve according to the manufacturer's instructions. Each experiment was repeated three times in duplicate.

MMP-9 activity assay. MMP-9 in the cell lysate was initially pulled down with mouse monoclonal anti-MMP9 antibody (sc-21733) (Santa Cruz Biotechnology, Inc.). MMP-9 activity was then measured with the SensoLyte 520 MMP-9 assay kit according to the manufacturer's instructions $(27,28)$. The supernatants were collected and incubated with 4-aminophenylmercuric acetate (AMPA) and MMP-9 substrate. The fluorescence intensity at Ex/Em wavelengths of 490/520 nm were used as a measure of MMP-9 activity. Each experiment was repeated three times in duplicate.

MTT cell proliferation assay. In vitro cell proliferation was determined with a MTT cell proliferation assay kit as described by the manufacturer (ATCC). Briefly, the cells were cultured at $15 \times 10^{3}$ cells/well in 96-well tissue culture plates and incubated at $37^{\circ} \mathrm{C}$ for $24 \mathrm{~h}$ with or without Ang-(1-7) (100 nM) treatment. At the end of the culture period, the cells were washed with PBS, the MTT reagents were added according to the manufacturer's instructions, and absorbance was measured at $570 \mathrm{~nm}$ using an ELISA plate reader. Each experiment was repeated three times in duplicate.

Statistical analysis. Statistical analysis was performed with SPSS for Windows 10.0 (SPSS, Inc., Chicago, IL, USA). Data values were presented as mean \pm SD. Comparisons of means among multiple groups were performed with one-way ANOVA followed by post-hoc pairwise comparisons using Tukey's tests. The correlation of protein levels of PODX and
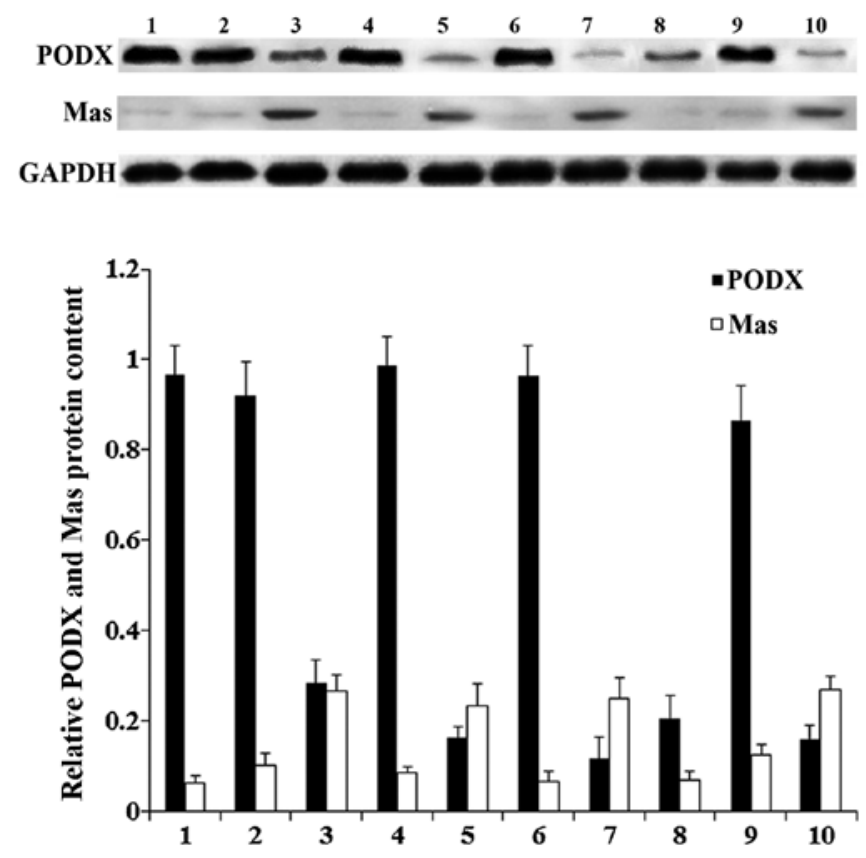

Figure 1. Protein levels of podocalyxin (PODX) and Mas in human glioblastoma multiforme (GBM) tumors. Protein levels of PODX and Mas in GBM tumor tissues from 10 consecutive patients (lanes 1-10) were determined with western blot analysis. Glyceraldehyde-3-phosphate dehydrogenase (GAPDH) blotting was used as a loading control. Density of the PODX and the Mas blots was normalized against that of the GAPDH blot to obtain a relative blot density to represent relative protein content. Three independent experiments were performed for each western blot analysis. Data values are presented as mean $\pm \mathrm{SD}$.

Mas were determined using Pearson's correlation analysis. A two-tailed $\mathrm{p}<0.05$ was considered to indicate a statistically significant result in the present study.

\section{Results}

Expression of PODX and Mas in human GBM tumor tissues. To investigate the relationship between PODX and Mas in human GBM tumors, we examined the expression of PODX and Mas in GBM tumor tissues from 10 consecutive patients. As shown in Fig. 1, the western blot analysis revealed that while PODX was normally highly expressed, Mas was generally expressed at a low level in human GBM tumors. Pearson's correlation analysis showed a strong negative correlation between the proteins levels of PODX and Mas in human GBM tumors $(\mathrm{r}=-0.768, \mathrm{p}<0.01)$.

Effects of PODX on the expression of Mas in GBM cells. We stably overexpressed PODX in LN-229 and U-118 MG human GBM cells by stable transfection, and on the other hand, stably transduced the cells with lentiviral shRNA to knock down PODX. We also stably expressed Mas in LN-229 and U-118 MG cells overexpressing PODX. In addition, as our pilot study suggested that PODX regulates Mas in GBM cells by a PI3Kdependent mechanism (data not shown), we included a selective PI3K inhibitor BKM120 in all the experiments in the present study (29). As shown in Fig. 2, compared with the controls, PODX was overexpressed $>3$-folds in LN-229 and $>2.5$-folds in U-118 MG cells. On the other hand, the endogenous PODX 

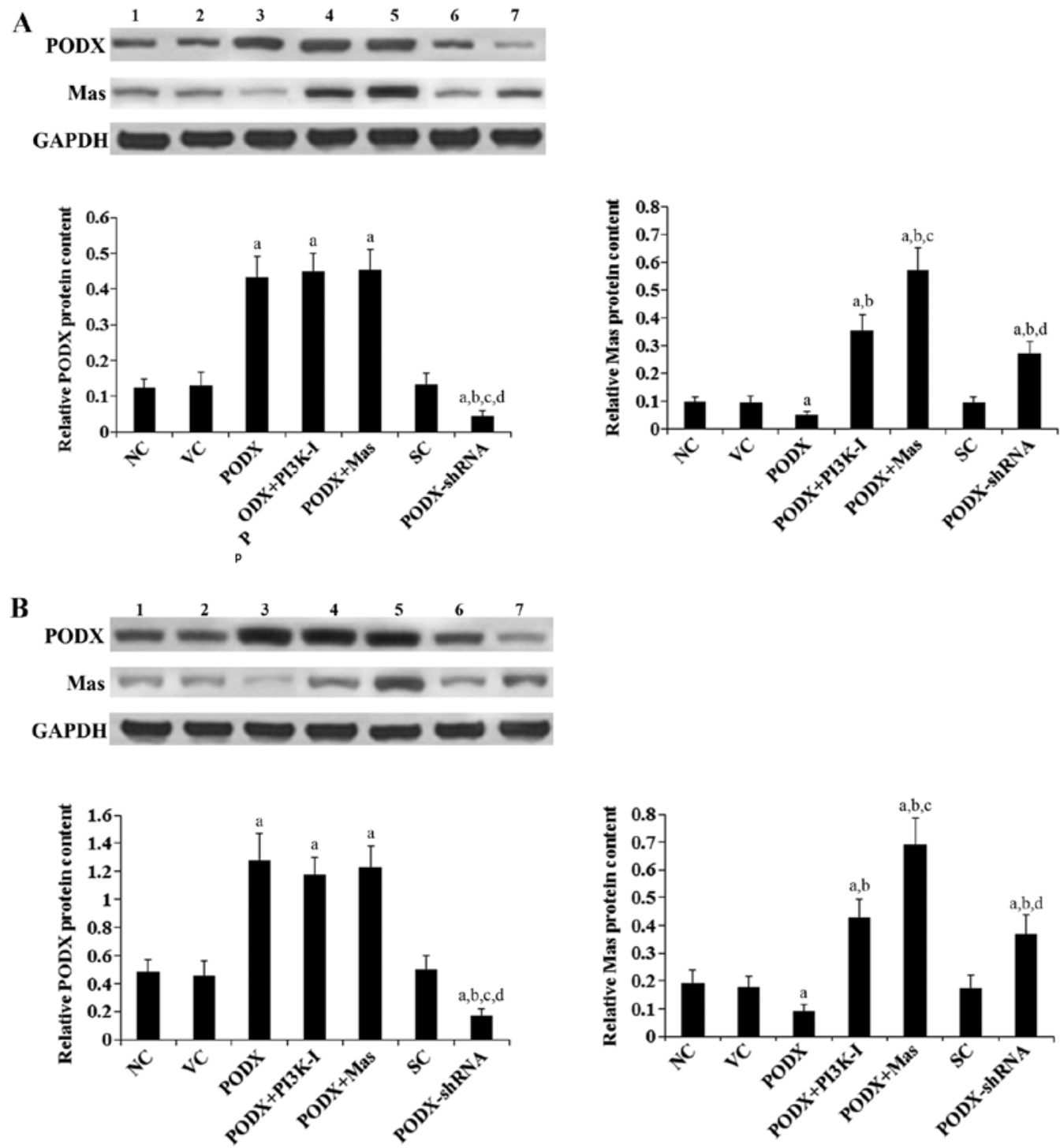

Figure 2. Protein levels of podocalyxin (PODX) and Mas in glioblastoma multiforme (GBM) cells with overexpression or knockdown of PODX. (A) In LN-229 and (B) U-118 MG human GBM cells, the protein levels of PODX and Mas were determined with western blot analyses in normal control cells (NC, lane 1), cells stably transfected with the empty pcDNA3.1 and the empty pIRESpuro3 vector (VC, lane 2), cells stably transfected with PODX (lane 3), cells stably transfected with PODX and treated with phosphatidylinositol 3-kinase (PI3K) inhibitor BKM120 (50 $\mu \mathrm{M})$ for $24 \mathrm{~h}$ (PODX+PI3K-I, lane 4), cells stably transfected with PODX and Mas (PODX+Mas, lane 5), cells stably transduced with scramble control shRNA (SC, lane 6), and cells stably transduced with PODX-shRNA (lane 7). Glyceraldehyde-3-phosphate dehydrogenase (GAPDH) blotting was used as a loading control. Density of the PODX and the Mas blots was normalized against that of the GAPDH blot to obtain a relative blot density to represent the relative protein content. Three independent experiments were performed for each western blot analysis. Data values are presented as mean $\pm \mathrm{SD}$. ${ }^{\mathrm{a}} \mathrm{P}<0.05$ vs. controls $(\mathrm{NC}, \mathrm{VC}$ and $\mathrm{SC})$; ${ }^{\mathrm{b}} \mathrm{p}<0.05 \mathrm{vs}$. PODX; ${ }^{\mathrm{c}} \mathrm{p}<0.05 \mathrm{vs}$. PODX+PI3K-I; ${ }^{\mathrm{d}} \mathrm{p}<0.05$ vs. PODX+Mas.

level was knocked down $~ 70$ and 55\% in LN-229 and U-118 MG cells, respectively. Overexpression of PODX decreased the Mas protein level by $\sim 50 \%$ in the $\mathrm{LN}-229$ and $\mathrm{U}-118 \mathrm{MG}$ cells. This effect was completely eliminated by selective PI3K inhibitor BKM120. On the other hand, knockdown of PODX increased the Mas protein level by $\sim 3$-folds in LN-229 cells and $\sim 2$-folds in U-118 MG cells (Fig. 2). Stable transfection of Mas increased the Mas protein level by $>6$-folds in LN-229 and $>3.5$-folds in U-118 MG cells, but had no significant effect on the expression of PODX (Fig. 2). BKM120 showed no significant effect on the protein level of PODX in both cell lines (Fig. 2). RT-qPCR (Fig. 3) and [25I]Ang-(1-7) binding assays (Fig. 4) showed similar data trends as in Fig. 2. Taken together, the results indicated that PODX downregulates the expression of Mas in GBM cells at the mRNA level through a PI3K-dependent mechanism, which leads to decreased density of Ang-(1-7)-binding Mas on the cell membrane of GBM cells.

Effects of PODX on Ang-(1-7)/Mas signaling in GBM cell invasion. To examine the effect of PODX on Ang-(1-7)/Mas signaling in GBM cell invasion, we performed in vitro cell invasion assays in GBM cells with or without Ang-(1-7) (100 nM) treatment for $24 \mathrm{~h}$. In the absence of Ang-(1-7), compared with the controls, overexpression of PODX increased cell invasion by $>3$-folds in LN-229 and $>2.3$-fold in U-118 MG cells; the effect was abolished by BKM120 (Fig. 5). By contrast, knockdown of PODX decreased cell invasion by $~ 50 \%$ in the two cell lines (Fig. 5). In the presence of Ang-(1-7) treatment, 

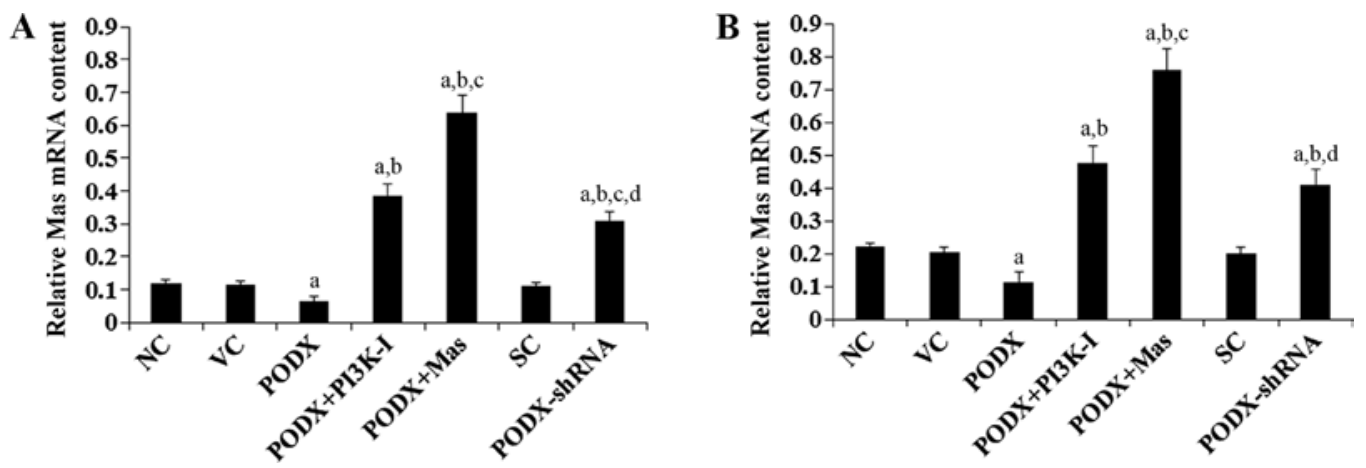

Figure 3. mRNA levels of Mas in glioblastoma multiforme (GBM) cells with overexpression or knockdown of PODX. (A) In LN-229 and (B) U-118 MG GBM cells, mRNA levels of Mas were determined with RT-qPCR in normal control cells (NC), cells stably transfected with the empty pcDNA3.1 and the empty pIRESpuro3 vector (VC), cells stably transfected with PODX, cells stably transfected with PODX and treated with phosphatidylinositol 3-kinase (PI3K) inhibitor BKM120 $(50 \mu \mathrm{M})$ for $24 \mathrm{~h}$ (PODX+PI3K-I), cells stably transfected with PODX and Mas (PODX+Mas), cells stably transduced with scramble control shRNA (SC), and cells stably transduced with PODX-shRNA. ${ }^{a} \mathrm{P}<0.05$ vs. controls (NC, VC and SC); ${ }^{\mathrm{b}} \mathrm{p}<0.05$ vs. PODX; ${ }^{\mathrm{c}} \mathrm{p}<0.05$ vs. PODX+PI3K-I; ${ }^{\mathrm{d}} \mathrm{p}<0.05$ vs. PODX+Mas.
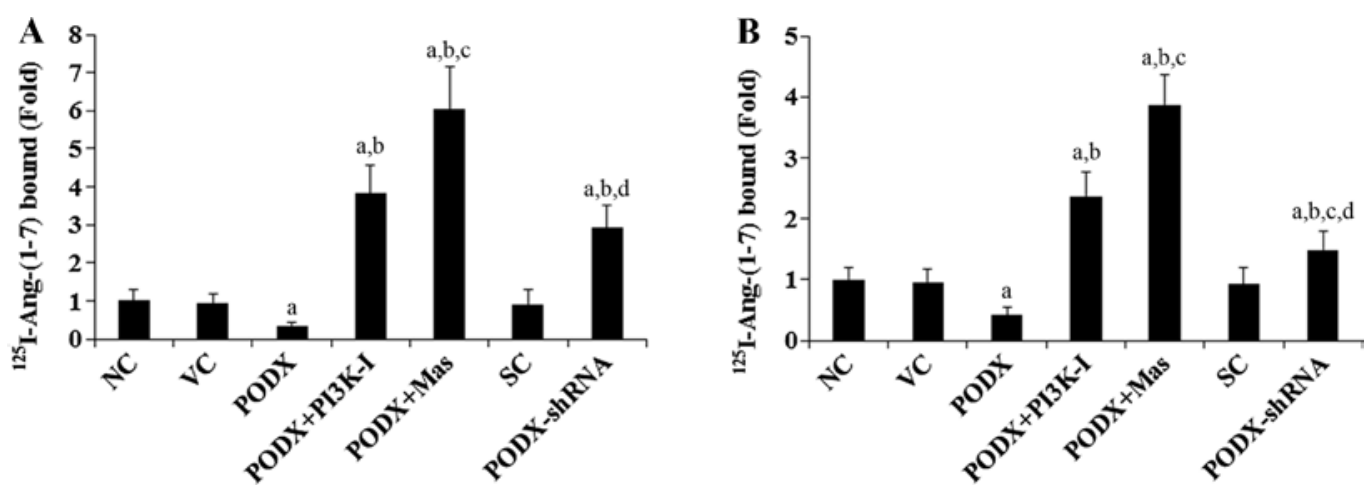

Figure 4. Saturation binding assay of Mas on the cell membrane of glioblastoma multiforme (GBM) cells with overexpression or knockdown of PODX. (A) In LN-229 and (B) U-118 MG GBM cells, saturation binding assays were conducted using increasing concentrations of ${ }^{125} \mathrm{I}-\mathrm{Ang}-(1-7)(1-22 \mathrm{nM})$ on the cell membranes of normal control cells (NC), cells stably transfected with the empty pcDNA3.1 and the empty pIRESpuro3 vector (VC), cells stably transfected with PODX, cells stably transfected with PODX and treated with phosphatidylinositol 3-kinase (PI3K) inhibitor BKM120 (50 $\mu \mathrm{M})$ for $24 \mathrm{~h}$ (PODX+PI3K-I), cells stably transfected with PODX and Mas (PODX+Mas), cells stably transduced with scramble control shRNA (SC), and cells stably transduced with PODX-shRNA. A single-site receptor binding model provided the best fit for data analysis. The disintegrations/min ( $\left.{ }^{\mathrm{d}} \mathrm{pm}\right)$ data were normalized against the cell number (per 20,000 cells) and shown as fold-changes to that of $\mathrm{NC}$ (designated as 1 ). ${ }^{\mathrm{a}} \mathrm{P}<0.05$ vs. controls (NC, VC and $\mathrm{SC}$ ); ${ }^{\mathrm{b}} \mathrm{p}<0.05 \mathrm{vs}$. PODX; ${ }^{\mathrm{p}} \mathrm{p}<0.05$ vs. PODX+PI3K-I; ${ }^{\mathrm{d}} \mathrm{p}<0.05$ vs. PODX+Mas.
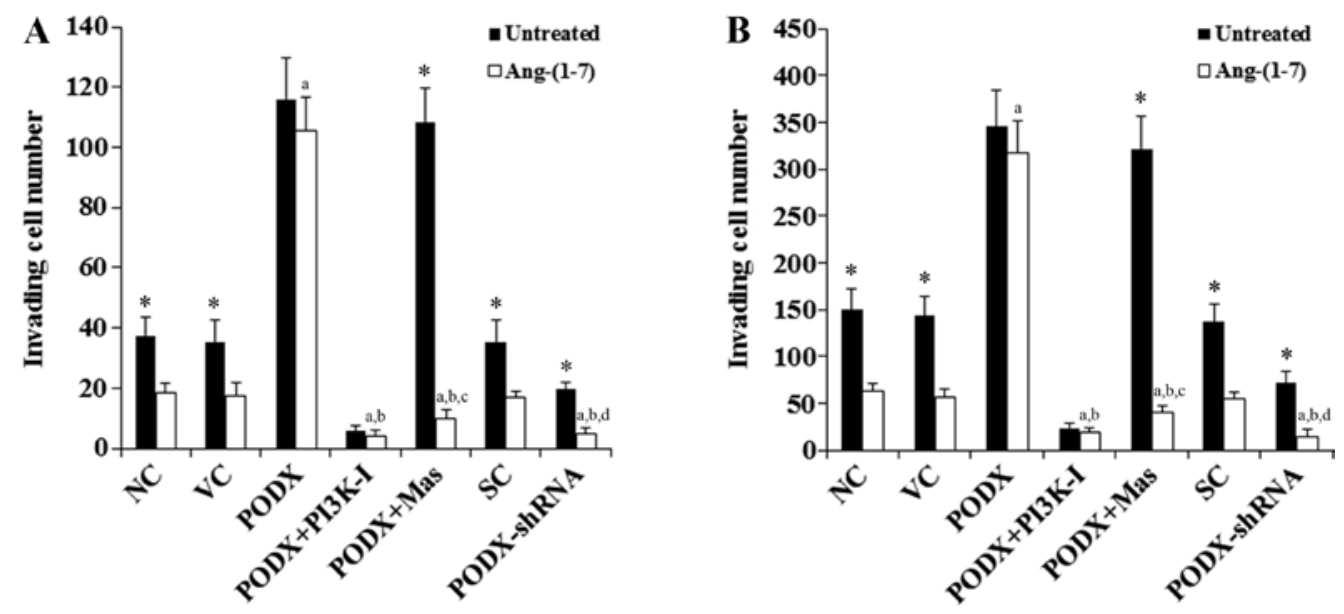

Figure 5. Effects of podocalyxin (PODX) on angiotensin (Ang)-(1-7)/Mas signaling on glioblastoma multiforme (GBM) cell invasion. In vitro cell invasion assays were performed with a Fluorimetric Cell Invasion Assay kit as described in Materials and methods in (A) LN-229 and (B) U-118 MG GBM cells. After $24 \mathrm{~h}$ of incubation with or without Ang-(1-7) (100 nM), invading cell numbers were determined by running a fluorescent cell dose curve in normal control cells (NC), cells stably transfected with the empty pcDNA3.1 and the empty pIRESpuro3 vector (VC), cells stably transfected with PODX, cells stably transfected with PODX and treated with phosphatidylinositol 3-kinase (PI3K) inhibitor BKM120 (50 $\mu \mathrm{M})$ for $24 \mathrm{~h}$ (PODX+PI3K-I), cells stably transfected with PODX and Mas (PODX+Mas), cells stably transduced with scramble control shRNA (SC), and cells stably transduced with PODX-shRNA. ${ }^{*} \mathrm{P}<0.05$ vs. Ang-(1-7). For cells treated with Ang-(1-7), ${ }^{\mathrm{a}} \mathrm{p}<0.05$ vs. controls (NC, VC and SC); ${ }^{\mathrm{b}} \mathrm{p}<0.05$ vs. PODX; ${ }^{\mathrm{c}} \mathrm{p}<0.05$ vs. PODX+PI3K-I; ${ }^{\mathrm{d}} \mathrm{p}<0.05$ vs. PODX+Mas. 
A
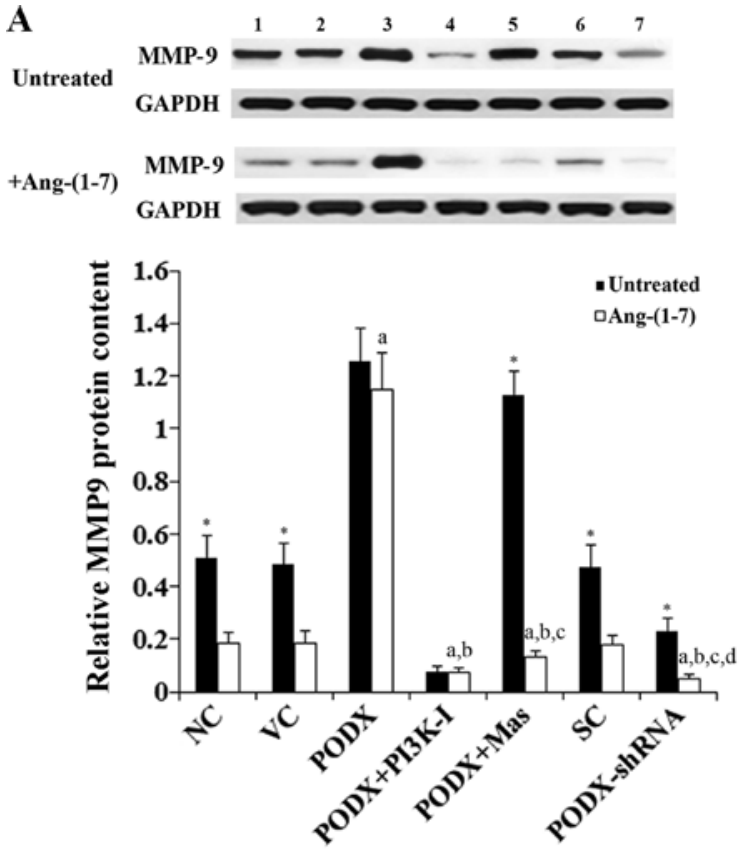

B
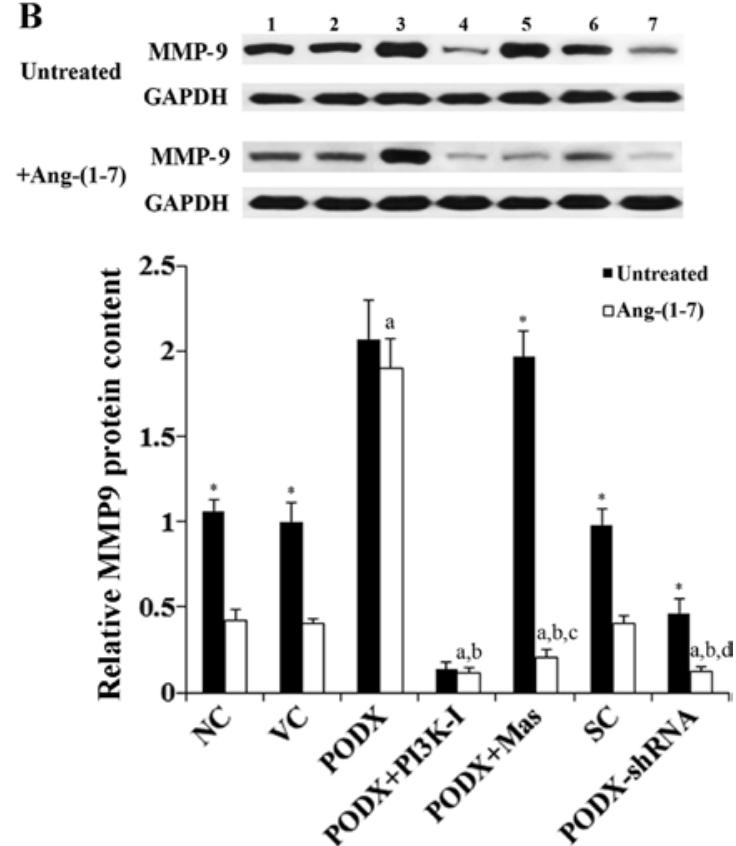

Figure 6. Effects of podocalyxin (PODX) on angiotensin (Ang)-(1-7)/Mas signaling in the expression of matrix metalloproteinase 9 (MMP9) in glioblastoma multiforme (GBM) cells. (A) In LN-229 and (B) U-118 MG GBM cells with or without Ang-(1-7) (100 nM) treatment for 24 h, the expression of MMP9 was determined with western blot analysis in normal control cells (NC, lane 1), cells stably transfected with the empty pcDNA3.1 and the empty pIRESpuro3 vector (VC, lane 2), cells stably transfected with PODX (lane 3), cells stably transfected with PODX and treated with phosphatidylinositol 3-kinase (PI3K) inhibitor BKM120 $(50 \mu \mathrm{M})$ for $24 \mathrm{~h}$ (PODX+PI3K-I, lane 4), cells stably transfected with PODX and Mas (PODX+Mas, lane 5), cells stably transduced with scramble control shRNA (SC, lane 6), and cells stably transduced with PODX-shRNA (lane 7). Glyceraldehyde-3-phosphate dehydrogenase (GAPDH) blotting was used as a loading control. Density of the MMP-9 blot was normalized against that of the GAPDH blot to obtain a relative blot density to represent relative MMP-9 content. Three independent experiments were performed for each western blot analysis. Data values are presented as mean \pm SD. "P<0.05 vs. Ang-(1-7). For cells treated with Ang-(1-7), ${ }^{\mathrm{a}} \mathrm{p}<0.05$ vs. controls (NC, VC and SC); ${ }^{\mathrm{b}} \mathrm{p}<0.05$ vs. PODX; ${ }^{\mathrm{c}} \mathrm{p}<0.05$ vs. PODX+PI3K-I; ${ }^{\mathrm{d}} \mathrm{p}<0.05$ vs. PODX+Mas.
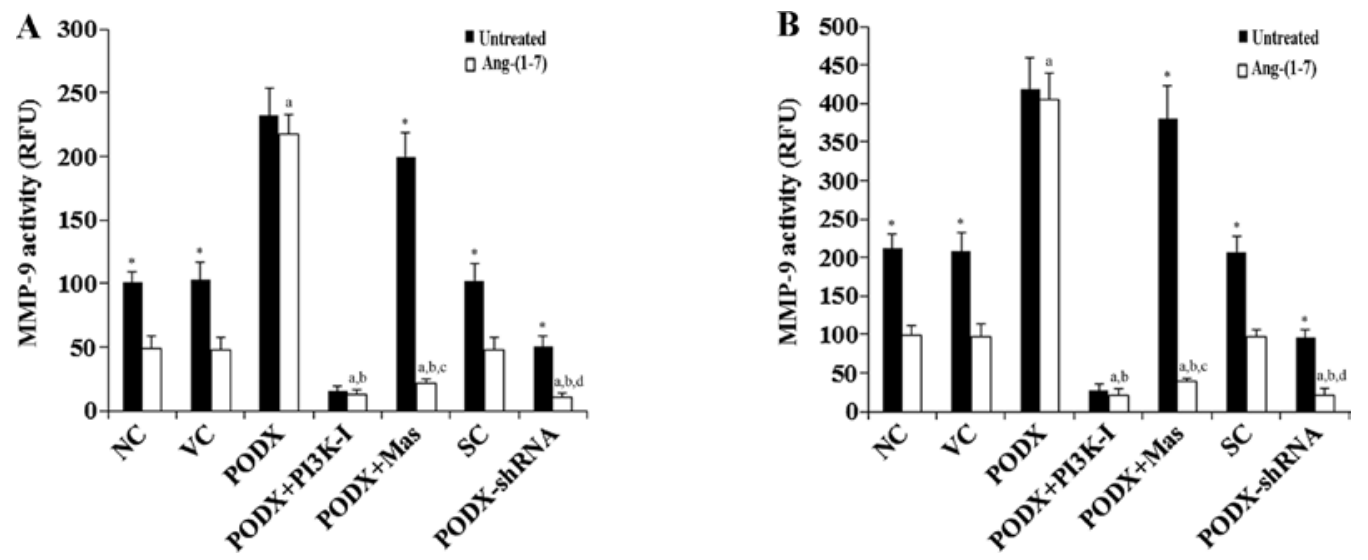

Figure 7. Effects of podocalyxin (PODX) on angiotensin (Ang)-(1-7)/Mas signaling in matrix metalloproteinase 9 (MMP-9) activities in glioblastoma multiforme (GBM) cells. (A) In LN-229 and (B) U-118 MG GBM cells with or without Ang-(1-7) (100 nM) treatment for 24 h, MMP-9 activities were determined with a SensoLyte 520 Fluorimetric MMP-9 Assay kit (AnaSpec) in normal control cells (NC), cells stably transfected with the empty pcDNA3.1 and the empty pIRESpuro3 vector (VC), cells stably transfected with PODX, cells stably transfected with PODX and treated with phosphatidylinositol 3-kinase (PI3K) inhibitor BKM120 $(50 \mu \mathrm{M})$ for $24 \mathrm{~h}$ (PODX+PI3K-I), cells stably transfected with PODX and Mas (PODX+Mas), cells stably transduced with scramble control shRNA (SC), and cells stably transduced with PODX-shRNA. The MMP-9 activity was shown in relative fluorescence units (RFU). ${ }^{*} \mathrm{P}<0.05$ vs. Ang-(1-7). For cells treated with Ang-(1-7), ${ }^{\mathrm{a}} \mathrm{p}<0.05$ vs. controls (NC, VC and SC); ${ }^{\mathrm{b}} \mathrm{p}<0.05$ vs. PODX; ${ }^{\mathrm{p}} \mathrm{p}<0.05$ vs. PODX+PI3K-I; ${ }^{\mathrm{d}} \mathrm{p}<0.05$ vs. PODX+Mas.

LN-229 and U-118 MG control cells showed $~ 50 \%$ decrease in cell invasion compared with those without Ang-(1-7) treatment, which was completely reversed by the overexpression of PODX (Fig. 5). On the other hand, in LN-229 and U-118 MG cells with knockdown of PODX and treated with Ang-(1-7), cell invasion was decreased by $>75 \%$ compared with those without Ang-(1-7) treatment (Fig. 5). In the absence of Ang-(1-7), the overexpression of Mas showed no significant effect on the promoting effect of PODX on LN-229 and U-118 MG cell invasion. However, in LN-229 and U-118 MG cells treated with Ang-(1-7), overexpression of Mas completely eliminated the promoting effect of PODX on cell invasion (Fig. 5). BKM120 completely eliminated the promoting effect of PODX on cell invasion in the two cell lines regardless of Ang-(1-7) treatment (Fig. 5), probably because the PI3K/Akt pathway is critical for the expression of MMPs and cancer cell invasion $(30,31)$. 

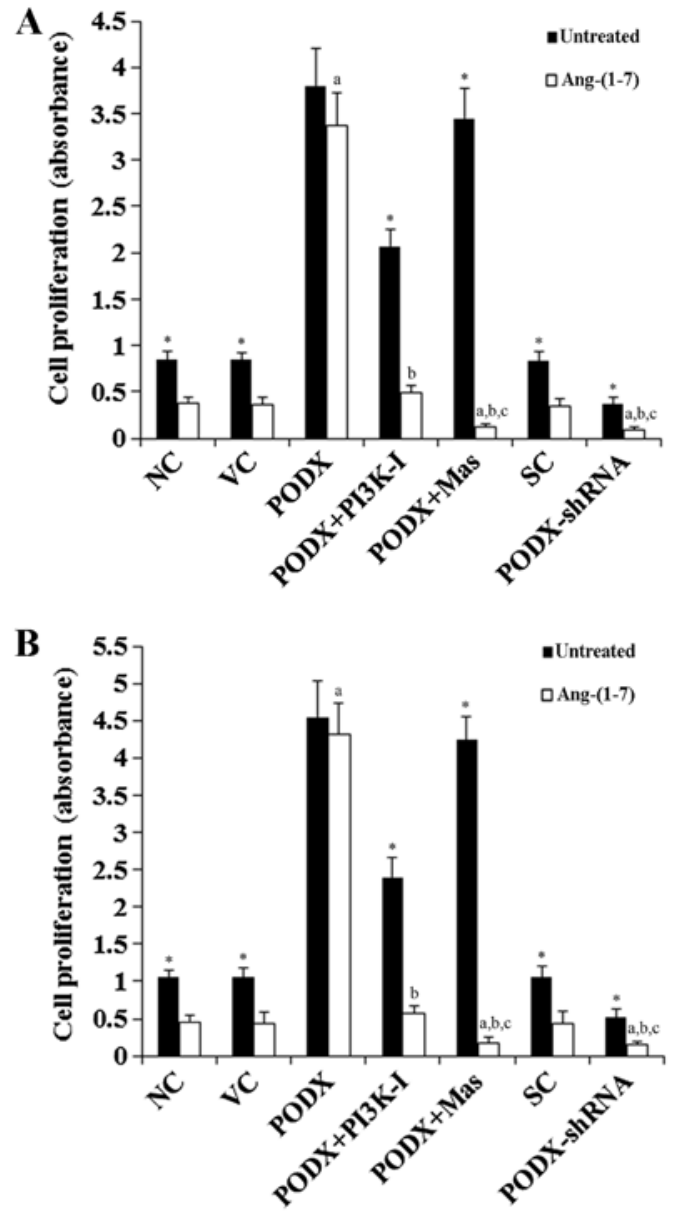

Figure 8. Effects of podocalyxin (PODX) on angiotensin (Ang)-(1-7)/Mas signaling in glioblastoma multiforme (GBM) cell proliferation. (A) In LN-229 and (B) U-118 MG GBM cells with or without Ang-(1-7) (100 nM) treatment for $24 \mathrm{~h}$, methylthiazoletetrazolium (MTT) cell proliferation assays were performed for $24 \mathrm{~h}$ in normal control cells (NC), cells stably transfected with the empty pcDNA3.1 and the empty pIRESpuro3 vector (VC), cells stably transfected with PODX, cells stably transfected with PODX and treated with phosphatidylinositol 3-kinase (PI3K) inhibitor BKM120 $(50 \mu \mathrm{M})$ for $24 \mathrm{~h}$ (PODX+PI3K-I), cells stably transfected with PODX and Mas (PODX+Mas), cells stably transduced with scramble control shRNA (SC), and cells stably transduced with PODX-shRNA. Cell proliferation at $24 \mathrm{~h}$ was shown in absorbance values at $570 \mathrm{~nm}$. ${ }^{*} \mathrm{P}<0.05$ vs. Ang-(1-7). For cells treated with Ang-(1-7), ${ }^{a} \mathrm{p}<0.05$ vs. controls (NC, VC and SC); ${ }^{b} \mathrm{p}<0.05$ vs. PODX; ${ }^{\mathrm{c}} \mathrm{p}<0.05$ vs. PODX+PI3K-I.

MMPs play a critical role in cancer cell invasion (32). Of the MMPs examined, we found that MMP-9 expression (Fig. 6) and activity (Fig. 7) in LN-229 and U-118 MG cells with or without Ang-(1-7) treatment showed a similar data trend as that in Fig. 5. Taken together, the results suggested that in the tumor environment where local RAS is generally present, antagonizing the inhibitory effect of Ang-(1-7)/Mas signaling is a major functional component of PODX in promoting the expression/activity of MMP-9 and invasion in GBM cells.

Effects of PODX on Ang-(1-7)/Mas signaling in GBM cell proliferation. To examine the effect of PODX on Ang-(1-7)/Mas signaling in GBM cell proliferation, we performed MTT cell proliferation assays in GBM cells with or without Ang-(1-7) (100 nM) treatment for $24 \mathrm{~h}$. In the absence of Ang-(1-7), compared with the controls, overexpression of
PODX increased cell proliferation $>4.3$-fold in LN-229 and U-118 MG cells, while the effect was decreased by $\sim 50 \%$ by BKM120. On the other hand, knockdown of PODX decreased cell proliferation by $>50 \%$ in the two cell lines (Fig. 8). In the presence of Ang-(1-7) treatment, LN-229 and U-118 MG control cells showed $\sim 55 \%$ decrease in cell proliferation compared with those without Ang-(1-7) treatment, which was completely reversed by the overexpression of PODX (Fig. 8). On the other hand, in LN-229 and U-118 MG cells with knockdown of PODX and treated with Ang-(1-7), cell proliferation was decreased by $\sim 75 \%$ compared with those without Ang-(1-7) treatment (Fig. 8). In the absence of Ang-(1-7), overexpression of Mas showed no significant effect on the promoting effect of PODX on LN-229 and U-118 MG cell proliferation. However, in LN-229 and U-118 MG cells treated with Ang-(1-7), overexpression of Mas completely eliminated the promoting effect of PODX on cell proliferation (Fig. 8). In the presence of Ang-(1-7) treatment, BKM120 decreased the promoting effect of PODX on cell proliferation by $\sim 85 \%$ in the two cell lines (Fig. 8). Taken together, the results suggested that in tumor environment where local RAS is generally present, antagonizing the inhibitory effect of the Ang-(1-7)/Mas axis through a PI3K-dependent mechanism is a major functional component of PODX in promoting GBM cell proliferation.

\section{Discussion}

Although there is evidence that PODX interacts with different mediators of metastasis, the role of PODX remains to be elucidated (12). Recent studies have shown that the local RAS system in tumor environment contributes significantly to tumor progression (14). As a counter-regulatory axis in RAS, Ang-(1-7)/Mas signaling has been shown to inhibit the growth and invasiveness of several human cancers, including $\operatorname{GBM}(14,23,33)$. Using human GBM tumor samples and human GBM cell models, we have demonstrated a crosstalk between PODX and Ang-(1-7)/Mas signaling in GBM cell invasion and proliferation. Two GBM cell lines were used as cell models in the present study: i) LN229, established from glioblastoma in the brain cortex of a 60-year-old female, showing epithelial morphology, and ii) U-118 MG established from glioblastoma of a 50-year-old male, showing mixed morphology. Similar findings in the two cell models with relatively great background differences demonstrate a generalizable role of the crosstalk between PODX and Ang-(1-7)/Mas signaling in GBM.

PODX is thought to regulate cell morphology and adhesion through its connections to intracellular proteins and to extracellular ligands (12). Ang-(1-7) is a biologically active member of the RAS (34). The physiological role of Ang-(1-7) was firmly established by two recent findings: i) identification of the ability of ACE2, an enzyme that generates Ang-(1-7) from Ang I or Ang II (33); ii) characterization of the $\mathrm{G}$ protein-coupled receptor Mas as a receptor that mediates the actions of Ang-(1-7) (35). In the present study, our results indicate that PODX downregulates the expression of Mas in GBM cells at the mRNA level, which leads to decreased density of Ang-(1-7)-binding Mas on the cell membrane of GBM cells. In addition, as a selective PI3K inhibitor readily abolished the inhibitory effect of PODX on the Mas mRNA level without significantly altering the expression of PODX, 
it suggests that PODX inhibits the expression of Mas by a PI3K-dependent mechanism at the transcription level in GBM cells. However, the underlying mechanisms remain to be determined in future studies.

PODX reportedly enhances invasion in many human cancers including GBM (9-11), while Ang-(1-7)/Mas signaling has been shown to inhibit the growth and invasiveness of several human cancers including GBM $(14,23,33)$. In the present study, overexpression of PODX significantly decreased the expression of Mas in GBM cells, which led to a markedly decreased density of Ang-(1-7)-binging Mas on the cell membrane. This result was in agreement with our findings that overexpression of PODX reversed the inhibitory effects of Ang-(1-7) on GBM cell invasion and proliferation. On the other hand, knockdown of PODX significantly increased the expression of Mas in GBM cells, which led to a markedly increased density of Ang-(1-7)-binging Mas on the cell membrane. This result was corroborated by our findings that knockdown of PODX significantly enhanced the inhibitory effects of Ang-(1-7) on GBM cell invasion and proliferation. Notably, while overexpression of Mas showed no significant effect on the promoting effect of PODX on GBM cell invasion and proliferation in the absence of Ang-(1-7), it completely eliminated the effect of PODX in the presence of Ang-(17). The findings suggest that inhibition of Ang-(1-7)/Mas signaling by downregulating the expression of Mas is a major functional component of PODX in the promotion of GBM cell invasion and proliferation.

MMPs play a critical role in cancer cell invasion (32). Of the MMPs examined, we found that the MMP-9 expression/activity was significantly altered by PODX and Ang-(1-7)/ Mas signaling. Our results suggest that PODX promotes GBM cell invasion mainly by antagonizing the inhibitory effect of Ang-(1-7)/Mas signaling on the expression/activity of MMP-9. A selective PI3K inhibitor completely abolished the promoting effect of PODX on the expression/activity of MMP-9 and GBM cell invasion regardless of Ang-(1-7) treatment. This result is in agreement with previous studies showing that the PI3K/Akt pathway is critical for the expression of MMPs and cancer cell invasion $(30,31)$.

Both PODX and Ang-(1-7)/Mas signaling are found in many types of cancers, including GBM $(5-11,14,23)$. While aberrant PODX expression has been found to be associated with increased aggressiveness in various types of cancer (9-10), Ang-(1-7)/Mas signaling has shown inhibitory effects on malignant progression $(14,23,33)$. The present study revealed that PODX is an endogenous antagonist of Ang-(1-7)/ Mas signaling in GBM, which suggests a novel functional role of PODX in malignant progression. The crosstalk between PODX and Ang-(1-7)/Mas signaling may also play important roles in the progression of other cancers besides GBM, which needs to be verified in future studies.

In conclusion, the present study has, to the best of our knowledge, provided the first evidence that PODX inhibits Ang-(1-7)/Mas signaling by dowregulating the expression of Mas through a PI3K-dependent mechanism in GBM cells. This effect leads to enhanced GBM cell invasion and proliferation. The results of the present study add new insights into the biological functions of PODX and the molecular mechanisms underlying GBM progression.

\section{Acknowledgements}

This study was supported by the Hunan Provincial Natural Science Foundation (grant no. 2014FJ4260), Hunan, China.

\section{References}

1. Zhang $\mathrm{Z}$ and Lin CC: Taking advantage of neural development to treat glioblastoma. Eur J Neurosci 40: 2859-2866, 2014.

2. Wen PY and Kesari S: Malignant gliomas in adults. N Engl J Med 359: 492-507, 2008.

3. Giese A, Bjerkvig R, Berens ME and Westphal M: Cost of migration: invasion of malignant gliomas and implications for treatment. J Clin Oncol 21: 1624-1636, 2003.

4. Nielsen JS and McNagny KM: The role of podocalyxin in health and disease. J Am Soc Nephrol 20: 1669-1676, 2009.

5. Riccioni R, Calzolari A, Biffoni M, et al: Podocalyxin is expressed in normal and leukemic monocytes. Blood Cells Mol Dis 37: 218-225, 2006.

6. Kelley TW, Huntsman D, McNagny KM, Roskelley CD and Hsi ED: Podocalyxin: a marker of blasts in acute leukemia. Am J Clin Pathol 124: 134-142, 2005.

7. Yasuoka H, Tsujimoto M, Hirokawa M, et al: Podocalyxin expression in undifferentiated thyroid carcinomas. J Clin Pathol 61: 1228-1229, 2008.

8. Hsu YH, Lin WL, Hou YT, et al: Podocalyxin EBP50 ezrin molecular complex enhances the metastatic potential of renal cell carcinoma through recruiting Racl guanine nucleotide exchange factor ARHGEF7. Am J Pathol 176: 3050-3061, 2010.

9. Sizemore S, Cicek M, Sizemore N, Ng KP and Casey G: Podocalyxin increases the aggressive phenotype of breast and prostate cancer cells in vitro through its interaction with ezrin. Cancer Res 67: 6183-6191, 2007.

10. Somasiri A, Nielsen JS, Makretsov N, et al: Overexpression of the anti-adhesin podocalyxin is an independent predictor of breast cancer progression. Cancer Res 64: 5068-5073, 2004.

11. Wu H, Yang L, Liao D, Chen Y, Wang W and Fang J: Podocalyxin regulates astrocytoma cell invasion and survival against temozolomide. Exp Ther Med 5: 1025-1029, 2013.

12. Kaprio T, Fermér C, Hagström J, Mustonen H, Böckelman C, Nilsson $\mathrm{O}$ and Haglund C: Podocalyxin is a marker of poor prognosis in colorectal cancer. BMC Cancer 14: 493, 2014.

13. De Gasparo M, Catt KJ, Inagami T, Wright JW and Unger T: International union of pharmacology. XXIII. The angiotensin II receptors. Pharmacol Rev 52: 415-472, 2000.

14. Ni L, Feng Y, Wan H, Ma Q, Fan L, Qian Y, Li Q, Xiang Y and Gao B: Angiotensin-(1-7) inhibits the migration and invasion of A549 human lung adenocarcinoma cells through inactivation of the PI3K/Akt and MAPK signaling pathways. Oncol Rep 27: 783-790, 2012.

15. George AJ, Thomas WG and Hannan RD: The renin angiotensin system and cancer: old dog, new tricks. Nat Rev Cancer 10: 745-759, 2010.

16. Röcken C, Röhl FW, Diebler E, Lendeckel U, Pross M, Carl-McGrath S and Ebert MP: The angiotensin II/angiotensin II receptor system correlates with nodal spread in intestinal type gastric cancer. Cancer Epidemiol Biomarkers Prev 16: 1206-1212, 2007.

17. Ferreira AJ, Murça TM, Fraga-Silva RA, Castro CH, Raizada MK and Santos RA: New cardiovascular and pulmonary therapeutic strategies based on the angiotensin-converting enzyme 2/angiotensin-(1-7)/mas receptor axis. Int J Hypertens 2012: 147825, 2012.

18. Raizada MK and Ferreira AJ: ACE2: a new target for cardiovascular disease therapeutics. J Cardiovasc Pharmacol 50: 112-119, 2007.

19. Der Sarkissian S, Huentelman MJ, Stewart J, Katovich MJ and Raizada MK: ACE2: a novel therapeutic target for cardiovascular diseases. Prog Biophys Mol Biol 91: 163-198, 2006.

20. Rice GI, Thomas DA, Grant PJ, Turner AJ and Hooper NM: Evaluation of angiotensin-converting enzyme (ACE), its homologue ACE2 and neprilysin in angiotensin peptide metabolism. Biochem J 383: 45-51, 2004.

21. Ferreira AJ and Santos RA: Cardiovascular actions of angiotensin-(1-7). Braz J Med Biol Res 38: 499-507, 2005.

22. Gomes ER, Santos RA and Guatimosim S: Angiotensin-(1-7)mediated signaling in cardiomyocytes. Int J Hypertens 2012: 493129, 2012. 
23. Garcia-Espinosa MA, Lesser GJ, Debinski W, Tallant EA and Gallagher PE: Angiotensin-(1-7), a peptide hormone with therapeutic potential for the treatment of glioblastomas (abstract). In: Proceedings of the 103rd Annual Meeting of the American Association for Cancer Research; 2012 Mar 31-Apr 4; Chicago, Illinois. Philadelphia (PA): AACR. Cancer Res 72 (Suppl 8): Abs. 1938, 2012. doi:1538-7445.AM2012-1938.

24. Gironacci MM, Adamo HP, Corradi G, Santos RA, Ortiz P and Carretero OA: Angiotensin (1-7) induces MAS receptor internalization. Hypertension 58: 176-181, 2011.

25. Wang B, Feng P, Xiao Z and Ren EC: LIM and SH3 protein 1 (Lasp1) is a novel p53 transcriptional target involved in hepatocellular carcinoma. J Hepatol 50: 528-537, 2009.

26. Feng Y, Hu J, Ma J, Feng K, Zhang X, Yang S, Wang W, Zhang J and Zhang Y: RNAi-mediated silencing of VEGF-C inhibits non-small cell lung cancer progression by simultaneously down-regulating the CXCR4, CCR7, VEGFR-2 and VEGFR3-dependent axes-induced ERK, p38 and AKT signalling pathways. Eur J Cancer 47: 2353-2363, 2011.

27. Jo YK, Park SJ, Shin JH, Kim Y, Hwang JJ, Cho DH and Kim JC: ARP101, a selective MMP-2 inhibitor, induces autophagyassociated cell death in cancer cells. Biochem Biophys Res Commun 404: 1039-1043, 2011.

28. Qazi H, Shi ZD and Tarbell JM: Fluid shear stress regulates the invasive potential of glioma cells via modulation of migratory activity and matrix metalloproteinase expression. PLoS One 6: e20348, 2011.

29. Fekete A, Bőgel G, Pesti S, Péterfi Z, Geiszt M and Buday L: EGF regulates tyrosine phosphorylation and membrane-translocation of the scaffold protein Tks5. J Mol Signal 8: 8, 2013.
30. Yang N, Hui L, Wang Y, Yang H and Jiang X: SOX2 promotes the migration and invasion of laryngeal cancer cells by induction of MMP-2 via the PI3K/Akt/mTOR pathway. Oncol Rep 31: 2651-2659, 2014

31. Yoon SO, Shin S, Lee HJ, Chun HK and Chung AS: Isoginkgetin inhibits tumor cell invasion by regulating phosphatidylinositol 3-kinase/Akt-dependent matrix metalloproteinase-9 expression. Mol Cancer Ther 5: 2666-2675, 2006.

32. Li Y, Liao Q, Li K, Zhong D, Weng X and Mi M: Knockdown of endothelin A receptor expression inhibits osteosarcoma pulmonary metastasis in an orthotopic xenograft mouse model. Mol Med Rep 5: 1391-1395, 2012.

33. Wegman-Ostrosky T, Soto-Reyes E, Vidal-Millán S and Sánchez-Corona J: The renin-angiotensin system meets the hallmarks of cancer. J Renin Angiotensin Aldosterone Syst: Aug 9, 2013 (Epub ahead of print).

34. Silva DM, Vianna HR, Cortes SF, Campagnole-Santos MJ, Santos RAS and Lemos VS: Evidence for a new angiotensin-(1-7) receptor subtype in the aorta of Sprague-Dawley rats. Peptides 28: 702-707, 2007.

35. Medeiros MA, França MS, Boileau G, Juliano L and Carvalho KM: Specific fluorogenic substrates for neprilysin (neutral endopeptidase, EC 3.4.24.11) which are highly resistant to serine- and metalloproteases. Braz J Med Biol Res 30: $1157-1162,1997$. 